\title{
Artigo/Article
}

\section{Parasitismo por Ascaris lumbricoides e seus aspectos epidemiológicos em crianças do Estado do Maranhão}

\author{
Parasitism due to Ascaris lumbricoides and its epidemiological characteristics among children \\ in the State of Maranhão
}

\section{Jefferson Conceição Silva ${ }^{1}$, Luis Fernando Viana Furtado ${ }^{2}$, Thaís Carvalho Ferro ${ }^{2}$, Kathlenn de Carvalho Bezerra ${ }^{2}$, Edésio Pereira Borges ${ }^{2}$ e Ana Carolina Fonseca Lindoso Melo ${ }^{3}$}

\begin{abstract}
RESUMO
Introdução: As enteroparasitoses representam um sério problema de saúde pública de cunho mundial. Essas afecções estão correlacionadas com condições precárias de saneamento básico aliado a ausência de noções básicas de higiene, observada, sobretudo na infância. Dentre esses parasitos, destaca-se o nematóide Ascaris lumbricoides, com alta incidência no Brasil e no mundo. A prevalência e a intensidade da infecção por esse patógeno foram analisadas mediante um estudo transversal em crianças residentes no município de Tutóia, no Estado do Maranhão, entre julho e dezembro de 2008. Métodos: A população do estudo foi constituída por crianças entre um a doze anos de idade, num total de 220 indivíduos. As amostras fecais foram recolhidas nos domicílios em frascos contendo solução conservadora (MIF) e processadas por meio da técnica de sedimentação espontânea. Com cada pai ou responsável pela criança foi aplicado um questionário padrão, cujos resultados foram utilizados para análise descritiva da amostra estudada. Resultados: A prevalência de infecção por A. lumbricoides foi de 53,6\%. A análise dos questionários revelou resultados alarmantes no que diz respeito ao grau de insalubridade ao qual a população está inserida, bem como seus precários hábitos de higiene. Conclusões: O índice de parasitoses no presente trabalho é um reflexo claro da falta de saneamento básico da região estudada, indicando um estado epidemiológico preocupante. Dessa forma, faz-se necessária uma política pública de conscientização e combate dessa patologia.
\end{abstract}

Palavras-chaves: Parasitas intestinais. Ascaris lumbricoides. Epidemiologia. Crianças.

\begin{abstract}
Introduction: Intestinal parasitoses are a serious public health problem of worldwide nature. These disorders are correlated with poor basic sanitation conditions coupled with lack of basic hygiene notions, and are observed especially among children. Among these parasites, the nematode Ascaris lumbricoides stands out, with high incidence in Brazil and worldwide. The prevalence and intensity of infection by this pathogen were analyzed by means of a cross-sectional study among children living in the municipality of Tutóia, State of Maranhão, between July and December 2008. Methods: The study population consisted of children between one and twelve years of age, totaling 220 individuals. Fecal samples were collected in households in vials containing MIF preserving solution and were processed using the spontaneous sedimentation technique. A standard questionnaire was applied to each parent or guardian, and the results were used for descriptive analysis on the study sample. Results: The prevalence of A. lumbricoides was $53.6 \%$. Analysis on the questionnaires revealed alarming results regarding the degree of unhealthy condition to which the population is subjected, in addition to its poor hygiene habits. Conclusions: The prevalence rate found in this study is a clear reflection of the lack of basic sanitation in the region studied. Thus, a public policy for raising awareness and combating this disease is needed.
\end{abstract}

Keywords: Intestinal parasites. Ascaris lumbricoides. Epidemiology. Children.

1. Universidade Estadual do Piauí, Parnaíba, PI. 2. Curso de Biomedicina, Universidade Federal do Piauí, Parnaíba, PI. 3. Departamento de Biomedicina, Universidade Federal do Piauí, Parnaíba, PI.

Endereço para correspondência: Dra Ana Carolina Fonseca Lindoso Melo. Depto Biomedicina/UFPI. Av. São Sebastião 2819, Campus Universitário Ministro Reis Veloso, 64202-020 Parnaíba, PI.

e-mail: carolinamelo@ufpi.br

Recebido para publicação em 05/05/2010

Aceito em 06/10/2010

\section{INTRODUÇÃO}

As enteroparasitoses representam um sério problema de saúde pública de cunho mundial ${ }^{1}$. No Brasil, essas doenças ocorrem nas diversas regiões do país, seja em zona rural ou urbana e em diferentes faixas etárias ${ }^{2-4}$. Essas afecções estão correlacionadas com níveis socioeconômicos mais baixos e condições precárias de saneamento básico, representando um flagelo, sobretudo para as populações mais pobres ${ }^{5}$.

Os principais parasitas relatados nessas pesquisas são Giardia lamblia, Entamoeba coli, Ascaris lumbricoides, Trichuris trichiura e Enterobius vermicularis $^{2,6,7}$. A maioria desses parasitas causa desnutrição, anemia, diarréia, obstrução intestinal e má absorção ${ }^{8}$.

No que se refere às doenças parasitárias intestinais, estas acometem principalmente crianças em idade escolar, o que pode comprometer seu desenvolvimento físico e intelectual ${ }^{9,10}$. Dentre essas parasitoses intestinais, destaca-se a ascaridíase, helmintíase de maior prevalência no mundo causada pelo nematóide Ascaris lumbricoides ${ }^{11}$. Em 2008, a Organização Mundial de Saúde (OMS) estimou que mais de 980 milhões de pessoas no mundo estariam parasitadas por esse agente.

A maioria das infecções por A. lumbricoides envolve pequeno número de parasitos adultos e é assintomática, diagnosticada em exames coproparasitológicos ou através da eliminação de parasitos nas fezes. A manifestação dos sintomas da ascaridíase depende do número de parasitos adultos albergados pelo indivíduo. Infecções maciças podem resultar em bloqueio mecânico do intestino delgado, principalmente em crianças ${ }^{11}$.

No presente trabalho, objetivou-se determinar a prevalência da ascaridíase em crianças do bairro São José, Cidade de Tutóia, Estado do Maranhão, bem como relatar seus possíveis fatores de risco, caracterizando descritivamente a epidemiologia da doença na região e nesta faixa etária. 


\section{MÉTODOS}

\section{Local}

O trabalho foi realizado durante o período de julho a dezembro de 2008, no bairro São José, localizado na zona noroeste do município de Tutóia, Estado do Maranhão, ocupando uma área de $427.489 \mathrm{~m}^{2}$, compreendendo 233 domicílios e aproximadamente 771 habitantes (Sistema de Informação de Atenção Básica - SIAB, 2007).

\section{Amostra}

A amostra estudada abrange 110 domicílios (47,2\% do total de municípios do bairro). De cada residência, obtiveram-se amostras fecais de duas crianças de 1 a 12 anos de idade, somando 220 indivíduos.

\section{Procedimento}

Aplicou-se em cada família um questionário padrão, baseado em um conjunto de questões objetivas e de múltipla escolha, levando-se em consideração a situação de saúde, higiene e saneamento básico.

As amostras fecais foram coletadas em potes específicos e acondicionadas a $4^{\circ} \mathrm{C}$. $\mathrm{O}$ método utilizado para análise foi o de sedimentação espontânea de Hoffman, Pons e Janer.

\section{Análise dos dados}

Este é um estudo epidemiológico do tipo transversal. A detecção da doença foi realizada através do exame parasitológico de fezes. A exposição ao risco foi determinada pela aplicação de questionários padrão. Os dados são apresentados em percentagens referentes à prevalência da doença e/ou fatores predisponentes ao desenvolvimento de enteroparasitoses. Foi realizada uma análise descritiva dos fatores de risco as parasitoses intestinais. As amostras de fezes e dados de exposição ao risco foram coletadas simultaneamente.

\section{Considerações Éticas}

O trabalho foi aprovado pelo Comitê de Ética da Universidade Federal do Piauí e os pais ou o responsável pela criança assinou um termo de consentimento autorizando a utilização do material coletado para fins de pesquisa.

\section{RESULTADOS}

O coeficiente geral de prevalência por $A$. lumbricoides foi de $53,6 \%$. A partir da análise dos questionários, pôde-se observar resultados alarmantes no que diz respeito ao grau de insalubridade ao qual a população está inserida, bem como seus precários hábitos de higiene.

No quesito saneamento básico, 78,1\% das famílias afirmaram realizar suas necessidades fisiológicas ao ar livre. Quanto ao destino do lixo, as famílias admitiram queimar $(75,5 \%)$, enterrar $(19,1 \%)$ ou jogar o lixo domiciliar em terrenos baldios (12,3\%) por não haver coleta periódica no bairro onde moram. No item proximidades a casa, observou-se a existência de lama e/ou água empoçada (3,6\%), lixo (12,5\%) e animais vadios $(38,2 \%)$. Todas as famílias afirmaram que em seus domicílios não havia a presença de pragas, todavia, admitiram ter algum tipo de animal.

Todas as famílias afirmaram lavar frutas e verduras antes do consumo. Destas, 80,9\% admitiu utilizar água advinda de poços manuais para beber e lavar roupas, as quais $71,8 \%$ afirmaram somente coar a água antes de beber, acreditando ser uma forma de tratamento eficaz. Quando interrogados sobre deslocamento para outras regiões, cerca de $80 \%$ afirmou não ter o hábito de viajar.
O histórico clínico revelou que 94,5\% das crianças não apresentavam sintomas, para verminoses, como prurido anal e perversão alimentar, enquanto que somente 1,3\% apresentaram diarréia, $0,8 \%$ vômito, e $3,2 \%$ dor abdominal. A pesquisa revelou também que $84,6 \%$ das crianças utilizavam medicação antiparasitária indicada por farmacêuticos ou balconistas, sem realizar exames coproparasitológicos. Ainda, $15,4 \%$ das crianças nunca fizeram o uso de qualquer medicação desse gênero.

No quesito higiene pessoal, todas as crianças tomavam três ou mais banhos por dia e possuíam o hábito constante de cortar as unhas. Em $65 \%$ das crianças observou-se o uso de calçados somente para sair, enquanto $25 \%$ usavam somente em casa, e $10 \%$ constantemente. Todas as famílias afirmaram que as crianças lavavam as mãos antes das refeições e após as excreções.

\section{DISCUSSÃo}

A prevalência por A. lumbricoides nesse trabalho é superior aos resultados de pesquisas realizadas no Espírito Santo ${ }^{12}$ e semelhantes aos resultados obtidos em Sergipe ${ }^{13}$. Nestes e em vários outros estudos realizados no Brasil, as condições higiênico-sanitárias se mostraram estritamente relacionadas com altas prevalências de parasitos intestinais ${ }^{14,15}$.

A análise dos questionários revelou que o bairro São José, em Tutóia, possui fatores que predispõem a disseminação de enteroparasitos. Tendo em vista essas precárias condições de saúde e saneamento básico, sugere-se que alguns indivíduos tenham sido parasitados na própria região onde moram, uma vez que grande parcela das famílias afirmou não se deslocar periodicamente para outras localidades, facilitando a reinfecção por $A$. lumbricoides.

Apesar de $100 \%$ das crianças consumirem frutas e verduras lavadas, a origem da água utilizada não garante que esses alimentos estejam isentos de contaminação ${ }^{16}$. O grau de contato com os alimentos já corresponde a um fator de risco, além disso, pesquisas demonstram que manipuladores de alimentos podem representar um potencial risco de transmissão de enteroparasitos caso não possuam cuidados básicos ${ }^{17,18}$.

As famílias entrevistadas mostraram um descomprometimento com a saúde quanto ao consumo e tratamento da água antes de beber. $O$ fato dessa parcela da população não fazer nenhum tipo de tratamento de água acaba por comprometer a sua saúde como um todo ${ }^{19}$. Komagome cols ${ }^{6}$, correlacionando a prevalência de enteroparasitoses em crianças com seus aspectos epidemiológicos, revelaram que o tipo de água utilizada para consumo foi um fator de risco detectado, uma vez que quem consumiu água não-filtrada apresentou 15,9 vezes mais chances de adquirir enteroparasitoses.

O histórico clínico revelou que a maioria das crianças não apresentava sintomas para ascaridíase. A sintomatologia da ascaridíase é bastante variável e nos quadros mais leves as manifestações são inespecíficas ${ }^{11}$. As infecções crônicas em crianças, ainda que sejam geralmente assintomáticas, podem produzir retardo de crescimento clinicamente significativo ${ }^{20,21}$. Alguns estudos apontam uma relação entre a infecção por $A$. lumbricoides e o surgimento da asma ${ }^{22,23}$.

A maioria das crianças do bairro que se apresentaram positivas para $A$. lumbricoides são indivíduos em idade escolar. No trabalho realizado por Basso $\mathrm{cols}^{24}$, com crianças nessa faixa etária, em $58 \%$ das fezes analisadas foi encontrado algum tipo de parasito intestinal, sendo mais prevalente a infestação por A. lumbricoides (47\%). Esses agentes podem exercer alto poder patogênico e influenciar no estado nutricional, no crescimento e na função cognitiva dos escolares ${ }^{25}$. 
Todas as famílias afirmaram ter algum tipo de animal em suas residências. Todavia, sabe-se que isso não foi fator determinante para a transmissão da ascaridíase, uma vez que esta não possui potencial zoonótico. Cerca de $100 \%$ das famílias também afirmaram não possuir pragas em casa. Descarta-se, portanto, a idéia de transmissão das enteroparasitoses através de vetores mecânicos dentro da própria residência.

No quesito higiene pessoal, todas as crianças mostraram hábitos de higiene saudáveis. Porém, o fato de apenas $10 \%$ usarem calçados constantemente leva a crer que isso pode ser um fator de risco para a transmissão de geohelmintos. O contato direto com o solo é um fator que facilita a transmissão desse tipo de parasitos ${ }^{26}$.

O fato de um percentual significativo das crianças nunca ter feito o uso de qualquer tipo de medicação antiparasitária leva a crer que a alta prevalência de ascaridíase se deve também ao grau de descomprometimento das famílias com a saúde da criança. Além disso, o difícil acesso ao sistema público de saúde traz consigo dificuldades para erradicação de doenças ${ }^{27}$.

Conclui-se que existe alta prevalência de $A$. lumbricoides nas crianças do bairro São José, em Tutóia, MA, indicando um estado epidemiológico preocupante. Desse modo, é nítida a necessidade da implementação de programas do governo que tentem reverter à questão das precárias condições higiênico-sanitárias do bairro em estudo.

Sabe-se que a educação em saúde para crianças é fator essencial para controle da ascaridíase, especialmente considerando as características da doença durante a infância: alta prevalência, alta porcentagem de resistência ao tratamento, altas taxas de eliminação de ovos e altos níveis de reinfecção. Portanto, sugere-se que de nada adianta o empenho do governo em reverter o quadro de elevada prevalência de ascaridíase em Tutóia se os moradores não adotarem medidas de educação preventiva, uma vez que a criança tem um papel importante na manutenção do ciclo do A. lumbricoides e que seus maus hábitos desempenham papel decisivo na disseminação dessas doenças.

\section{CONFLITO DE INTERESSE}

Os autores declaram não haver nenhum tipo de conflito de interesse no desenvolvimento do estudo.

\section{REFERÊNCIAS}

1. Mamus CNC, Moitinho ACC, Grube CC, Melo EM, Weiler EB, Abreu CA, et al. Enteroparasitoses em um centro de educação infantil do município de Iretama/ PR. SaBios Rev Saude Biol 2008; 3:39-44.

2. Monteiro AMC, Silva EF, Almeida KS, Sousa JJN, Mathias LA, Baptista F, et al. Parasitoses intestinais em crianças de creches públicas localizadas em bairros periféricos do munícipio de Coari, Amazonas, Brasil. Rev Patol Trop 2009; 38:284-290.

3. Hurtado-Guerrero AF, Alencar FH, Hurtado-Guerrero JC. Ocorrência de enteroparasitas na população geronte de Nova Olinda do Norte - Amazonas. Acta Amaz 2005; 35:487-490.

4. Fonseca EOL, Teixeira MG, Barreto ML, Carmo EH, Costa MCN. Prevalência e fatores associados às geo-helmintíases em crianças residentes em municípios com baixo IDH no Norte e Nordeste brasileiros. Cad Saude Publica 2010; 26:143152.

5. Grillo LP, Carvalho LR, Silva AC, Verreschi ITN, Sawaya AL. Influência das condições socioeconômicas nas alterações nutricionais e na taxa de metabolismo de repouso em crianças escolares moradoras em favelas no município de São Paulo. Rev Assoc Med Brasil 2000; 46:7-14.
6. Komagome SH, Romagnoli MPM, Previdelli ITS, Falavigna DLM, Dias MLGG, Gomes ML. Fatores de risco para infecção parasitária intestinal em crianças e funcionários de creche. Cienc Cuid Saude 2007; 6:442-447.

7. Silva LP, Silva RMG. Ocorrência de enteroparasitos em centros de educação infantil no município de Patos de Minas, MG, Brasil. Biosci J 2010; 26:147-151.

8. Assis M, Borges FP, Santos RCV, Lunardelli A, Gaspareto PB, Graziottin CM, et al. Prevalência de enteroparasitos em moradores de vilas periféricas de Porto Alegre, RS. Rev Bras Anal Clin 2003; 35:215-217.

9. Ogliari TCC, Passos JT. Enteroparasitas em estudantes de quintas séries do Colégio Estadual de Terra Boa, ampina Grande do Sul, Paraná (Sul do Brasil). Acta Biol Par 2002; 331:65-70.

10. Saturnino ACRD, Nunes JFL, Silva EMA. Relação entre ocorrência de parasitas intestinais e sintomatologia observada em crianças de uma comunidade carente de Cidade Nova, em Natal - Rio Grande do Norte, Brasil. Rev Bras Anal Clin 2003; 35:85-87.

11. Melo MCB, Klem VGQ, Mota JAC, Penna FJ. Parasitoses intestinais. Rev Med 2004; 14:3-12.

12. Castro AZ, Viana, JDC, Peredo AA, Donatele DM. Levantamento das parasitoses intestinais em escolares da rede pública na cidade de Cachoeiro de Itapemirim - ES. Newslab 2004; 64:140-144.

13. Pereira CW, Santos FN. Prevalência de geo-helmintíases em crianças atendidas na rede pública de saúde de Neópolis, município do estado de Sergipe. Rev Bras Anal Clin 2005; 37:111-114.

14. Baptista SC, Breguez JMM, Baptista MCP, Silva GMS, Pinheiro RO. Análise da incidência de parasitoses intestinais no município de Paraíba do Sul, RJ. Rev Bras Anal Clin 2006; 38: 271-273.

15. Silva MCM, Monteiro CSP, Araújo BAV, Silva JV, Povoa MM. Determinação da infecção por Entamoeba histolytica em residentes da área metropolitana de Belém, Pará, Brasil, utilizando ensaio imunoenzimático (ELISA) para detecção de antígenos. Cad Saude Publica 2005; 21:969-973.

16. D’Aguila PS, Roque OCC, Miranda CAS, Ferreira AP. Avaliação da qualidade de água para abastecimento público do Município de Nova Iguaçu. Cad Saude Publica 2000; 16:791-798.

17. Nolla AC, Cantos GA. Relação entre a ocorrência de enteroparasitoses em manipuladores de alimentos e aspectos epidemiológicos em Florianópolis, Santa Catarina, Brasil. Cad Saude Publica 2005; 21:641-645.

18. Silva JO, Capuano DM, Takayanagui OM, Júnior EG. Enteroparasitoses e onicomicoses em manipuladores de alimentos do município de Ribeirão Preto, SP, Brasil. Rev Bras Epidemiol 2005; 8:385-392.

19. Dias MLGG, Souza D, Nishi L, Pupulin ART, Guilherme ALF, Falavigna DLM. Avaliação da água do sistema de abastecimento municipal de Maringá, PR, com relação à possível ocorrência de Cryptosporidium sp. e Giardia sp. Cienc Cuid Saude 2008; 7:100-106.

20. Becker AA, Ioschpe R, Delwing D, Canali J. Incidência de parasitoses intestinais em escolares do município de Novo Hamburgo-RS. Rev Bras Anal Clin 2002; 34:85-87.

21. Ferreira P, Lima MR, Oliveira FB, Pereira MLM, Ramos LBM, Marçal MG, et al. Ocorrência de parasitas e comensais intestinais em crianças de escola localizada em assentamento de sem-terras em Campo Florido, MG, Brasil. Rev Soc Bras Med Trop 2003; 36:109-111.

22. Silva MTN, Andrade J, Tavares-Neto J. Asma e ascaridíase em crianças de 2 a 10 anos de um bairro de periferia. J Ped 2003; 79:227-232.

23. Cunha AJLA. Asma e ascaridíase: persiste a controvérsia. J Ped 2003; 79:199200.

24. Basso RMC, Silva-Ribeiro RT, Soligo DS, Ribacki SI, Callegari-Jacques SM, Zoppas BCA. Evolução da prevalência de parasitoses intestinais em escolares em Caxias do Sul, RS. Rev Soc Bras Med Trop 2008; 41:263-268.

25. Roque FC, Borges FK, Signori LGH, Chazan M, Pigatto T, Coser TA, et al. Parasitos Intestinais: Prevalência em Escolas da Periferia de Porto Alegre - RS. NewsLab 2005; 69:152-162.

26. Alves JR, Macedo HW, Ramos Jr AN, Ferreira LF, Gonçalves MLC, Araújo A. Parasitoses intestinais em região semi-árida do Nordeste do Brasil: resultados preliminares distintos das prevalências esperadas. Cad Saude Publica 2003; 19:667-670.

27. Silva E, Sarreta FO, Bertani IF. As políticas públicas de saúde no Brasil: o Sistema Único de Saúde (SUS) e a rede de saúde em Franca. Serv Soc Real 2007; 16:81-103. 